\title{
Evaluation de la disponibilité saisonnière du fourrage ligneux en zone soudanienne du Tchad: cas du terroir de N'Guetté 1
}

\author{
A. B. BECHIR ${ }^{1 *}$, L. Y. MOPATE ${ }^{1}$ et C. Y. KABORE-ZOUNGRANA ${ }^{2}$ \\ ${ }^{I}$ Laboratoire de Recherches Vétérinaires et Zootechniques (LRVZ), BP 433, N'Djaména (Tchad) / Pôle \\ Régional de Recherche Appliquée au Développement des Systèmes Agricoles d'Afrique Centrale (PRASAC), \\ www.prasac-cemac.org (235) 25270 24/25 ; fax : (235) 527877. \\ ${ }^{2}$ Laboratoire d'Etudes et de Recherches des Ressources Naturelles et des Sciences de l'Environnement \\ (LERNSE), Université Polytechnique de Bobo-Dioulasso (UPB) (Burkina Faso), 01 B.P. 1091, Bobo- \\ Dioulasso 01,Tel : (00226) 209806 35,E-mail : cykabore@yahoo.fr \\ *Auteur correspondant,Tel. : (235)6254261,E-mail :ali_brabe@yahoo.fr
}

\section{RESUME}

L'évaluation de la disponibilité saisonnière du fourrage ligneux en zone soudanienne du Tchad a été menée dans le terroir de N'Guetté 1. L'objectif de cette étude a été d'analyser les variations saisonnières des ressources fourragères ligneuses dans ce terroir. La méthode de sondage systématique a été utilisée pour la description de la végétation. Les mesures ont été faites par une méthode dérivée de la méthode de relevé phytosociologique, adaptée aux espèces arborescentes. Les relevés ont été effectués dans 12 strates, durant les quatre saisons liées au calendrier fourrager. Les espèces les plus représentées étaient: Anogeissus leiocarpa, Combretum collinum et Pterocarpus erinaceus. L'échantillonnage a montré une variation dans la contribution des espèces au niveau des 12 strates. Le nombre de contacts maximal d'espèces a été enregistré en saison des pluies, dans les strates les plus basses $(2$ et $1 \mathrm{~m}$ ). Les strates inférieures ont été dominées par Combretum collinum, alors qu'au niveau des strates moyennes et supérieures, le plus grand nombre de présence est obtenu avec Pterocarpus erinaceus et Danielia oliveri. Une diminution de contribution spécifique est observée pendant la saison sèche chaude pour l'ensemble des espèces. Afin de permettre la régénération des espèces fourragères ligneuses les plus utilisées dans la complémentation des animaux en saison sèche, la mise en repos temporaire du terroir a été proposée.

(c) 2009 International Formulae Group. All rights reserved.

Mots clés : élevage, pâturage aérien, fourrages ligneux, strate, Tchad.

\section{INTRODUCTION}

$\mathrm{Au}$ Tchad, les pâturages naturels représentent pour l'élevage extensif la ressource la plus importante sur le plan alimentaire exploitable par les ruminants (Gaston, 1981). Cet élevage extensif valorise l'ensemble des ressources fourragères souvent hétérogènes dans l'espace et très variables dans le temps. Cette variabilité est importante, et concerne aussi bien la quantité et la qualité $\mathrm{du}$ fourrage produit que les saisons de leur production. Il en résulte une incertitude dans la prévision des apports alimentaires et des risques importants de disettes périodiques. Le disponible fourrager fluctue souvent dans des limites larges avec une alternance de phase d'excédents et de phase de pénurie plus ou moins sévère. Les fluctuations saisonnières en quantité comme en qualité des disponibilités fourragères, ne permettent pas un équilibre entre la densité du cheptel et les ressources fourragères (Hiernaux et Le Houerou, 2006). Les périodes et les amplitudes de variation des productions fourragères des parcours conditionnent l'organisation de la conduite des troupeaux, le rythme des déplacements 
des animaux et donc la gestion de l'espace pastoral et territorial (Hiernaux et al., 1994). La complémentation du fourrage herbacé à base des ligneux, accessoire pendant une partie de l'année, devient indispensable en saison sèche chaude pour l'entretien des animaux. La plupart des espèces ligneuses conservent des feuilles vertes toute l'année ou pendant une partie (Hiernaux et al., 1994 ; Hiernaux, 2000). Plusieurs études ont mis l'accent sur l'importante variabilité des ressources et surtout le rôle des espèces ligneuses dans les bilans fourragers des systèmes d'élevage des zones semi-arides d'Afrique (Hiernaux, 1980, Le Houerou, 1980 ; Piot, 1980 ; Sacko, 1991). En dépit de leur importance dans l'alimentation du bétail, les ligneux sont rarement comptabilisés parmi les ressources fourragères et lorsqu'ils le sont, il n'est pas tenu compte de leurs variations (Breman et De Ridder, 1991). L'objectif de cette étude a été d'évaluer la disponibilité saisonnière des fourrages ligneux des parcours du terroir de N'Guétté 1, afin de permettre une meilleure gestion.

\section{MATERIELS ET METHODES \\ Milieu de l'étude}

Le terroir de N'Guétté 1 est situé à 40 $\mathrm{km}$ à l'Est de la ville de Pala dans le Sud Ouest du Tchad, entre $15^{\circ} 11^{\prime} 44^{\prime}$ ' et $15^{\circ} 15^{\prime}$ $40^{\prime}$ ' de longitude Est, et $9^{\circ} 17^{\prime} 46^{\prime}$ ' et $9^{\circ} 24^{\prime}$ 01" de latitude Nord. Il couvre une superficie de 5280 ha et s'étend sur $15 \mathrm{~km}$ du Nord au sud et 9 km d'Est à l'Ouest (Figure 1).

Le relief est constitué des plateaux s'élevant de 300 à $550 \mathrm{~m}$, faiblement ondulés et entaillés de vallées qui représentent les contreforts du massif Yadé au Sud et débouchent au Nord sur la plaine du MayoKebbi (Cabot, 1965). Le climat est de type soudanien avec une saison des pluies (avril à octobre) et une saison sèche (novembre à mars). La pluviosité moyenne annuelle est de $1200 \mathrm{~mm}$ et la température annuelle moyenne varie entre 22 et $35^{\circ} \mathrm{C}$. En réalité, les différents types de temps se succèdent au cours de l'année de la manière suivante :

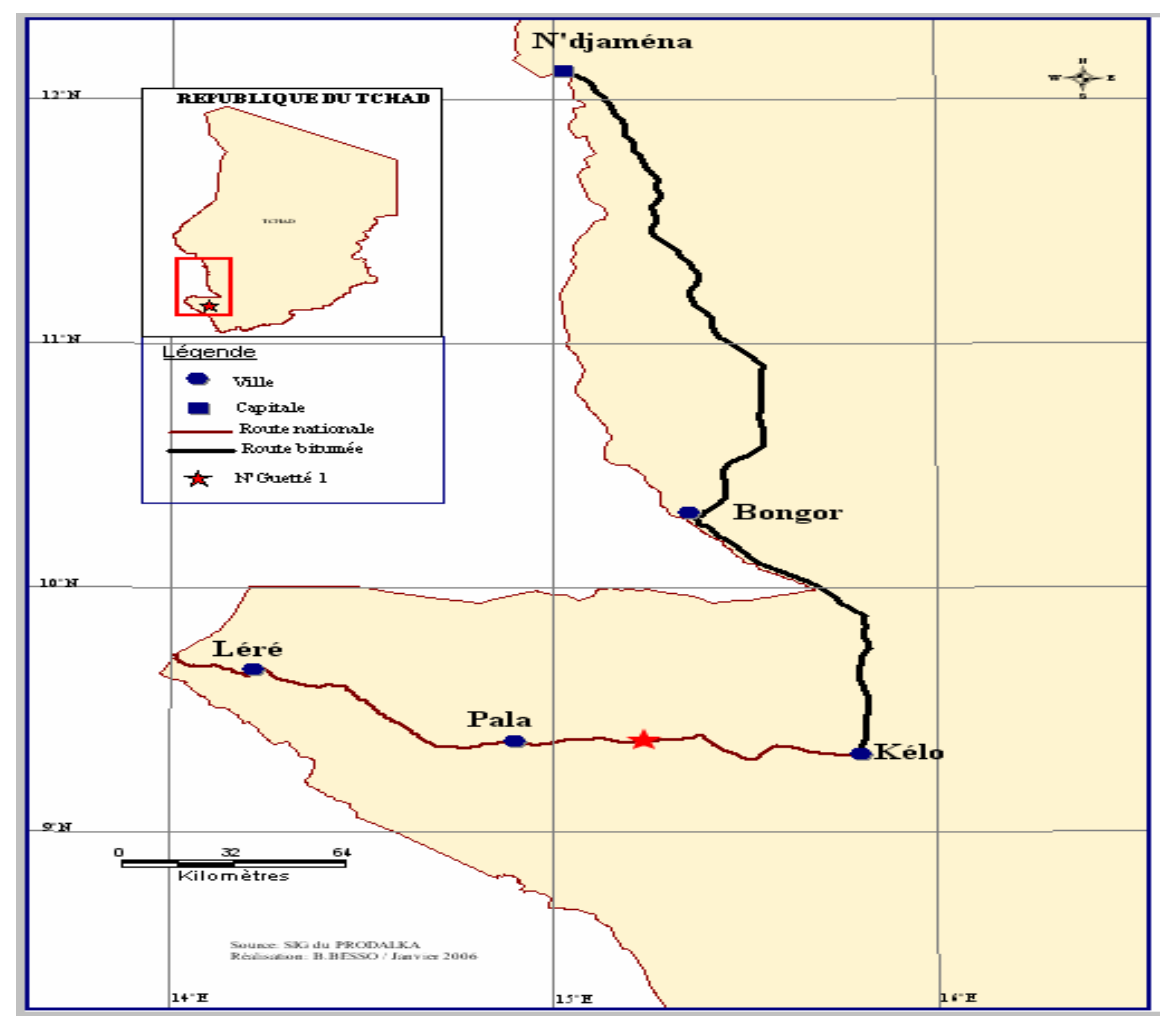

Figure 1: Localisation de la zone d'étude. 
- de novembre à mars: les hautes pressions continentales et l'harmattan de Nord-Est dominent. Il y a persistance des brumes sèches ; l'hygrométrie est très basse et on note une évaporation intense ;

- de mars à avril: la mousson du SudOuest glisse son extrémité en biseau sous la masse continentale dans la partie septentrionale $\mathrm{du}$ pays. Les premières tornades sèches et les premières pluies apparaissent. Puis cette première zone d'instabilité progresse du Sud au Nord ;

- d'avril à juin : le flux de mousson atteint son épaisseur maximal et occasionne la formation des foyers orageux isolés ;

- de juin à septembre: il y a une diminution des pluies en fréquence et en quantité au Nord et un retrait rapide de la masse d'air humide vers le Sud ;

- en octobre : quelques nuages marqués par la faible épaisseur du flux de mousson persistent ;

- en fin octobre : un vent de secteur Nord à Nord-Est s'installe sur la majeure partie du pays.

Ce schéma descriptif et classique des différents types de temps dans l'année correspond bien à celui des éleveurs tchadiens qui s'appuient sur un découpage de l'année en 5 saisons présenté dans le tableau 1. La saison des pluies en un lieu donné coïncide sensiblement avec l'arrivée du Front Inter Tropical (FIT) en provenance des régions côtières et la fin des pluies, à son retour vers la côte.

Dans le cadre du présent travail, nous n'avons retenu que quatre (4) saisons ; la cinquième, début de saison des pluies est très courte et se trouve ainsi confondue avec la fin de la saison sèche chaude.

\section{Choix des stations d'étude}

La représentativité en termes de superficie et de type de végétation et l'état de la formation (signes visibles de fréquentation et de pâture par les animaux) ont été les principaux critères qui ont guidé au choix des cinq stations d'étude. En raison de l'état d'homogénéité physionomique et floristique de certaines unités de végétation, le nombre de lignes d'observation par station a varié entre un et cinq.

\section{Mise en place du dispositif}

Les relevés de végétation ont été rigoureusement effectués sur les mêmes sites. Afin de faciliter leur localisation précise, les lignes d'observations ont été matérialisées par des repères fixes, constitués des tuyaux de 20 $\mathrm{cm}$ de long, fixés au sol à l'aide du béton. Les tuyaux ne dépassent le niveau du sol que de 2 ou $3 \mathrm{~cm}$. Les piquets amovibles, entre lesquels a été tendu le double décamètre, ont été plantés dans ces tuyaux. Afin de retrouver plus aisément l'emplacement des lignes, un ou deux gros arbres marqués à la peinture rouge et appartenant à des espèces différentes de celles qui sont dominantes sur la station ont été choisis comme repère de base. Les coordonnées GPS du point marquant le début de la ligne et celles des repères de base ont été également relevées.

\section{Mesures sur les stations : échantillonnage et relevés}

Les relevés de terrain ont été effectués par la méthode de sondage systématique utilisée par Ouedraogo (2006) dans l'étude de la distribution et de la structure des peuplements ligneux au Burkina Faso. La structure des populations a été évaluée à partir d'échantillonnages aléatoires sur des parcelles unitaires de $50 \mathrm{~m}$ x $25 \mathrm{~m}$. Dans ces parcelles, l'inventaire a concerné les arbres, arbustes et lianes dont le diamètre à $1,30 \mathrm{~m}$ du sol $(\mathrm{D} 1,30)$ est supérieur ou égal à $5 \mathrm{~cm}$. La circonférence du tronc à $1,3 \mathrm{~m}$ de hauteur (D1, 3) a été mesurée avec un ruban gradué, qui selon Rondeux (1999) donne des valeurs moins ambiguës que la mesure du diamètre. Le diamètre (D) a été ensuite calculé par la formule $: \mathrm{D}=$ circonférence $/ \pi$. A l'intérieur de ces mêmes parcelles, des sous-parcelles de $5 \mathrm{~m}$ x $5 \mathrm{~m}$ ont été installées pour étudier la régénération. Dans chaque sous-parcelle, tous les sujets dont le diamètre à $1,30 \mathrm{~m}$ du sol est inférieur à $5 \mathrm{~cm}$ sont considérés comme jeunes individus. Les mesures de fréquences le long d'une ligne de $50 \mathrm{~m}$ ont été effectuées grâce à une méthode dérivée de la méthode de points quadrats alignés (Daget et Poissonet, 1971), mais adaptée aux espèces arborescentes (César et Zoumana, 1999). L'écart entre deux points de lecture a été de 1 m. Des perches télescopiques emboîtables, en tube d'aluminium de $1,50 \mathrm{~m}$ de long, 
Tableau 1: Caractéristiques climatiques des saisons selon les agro-éleveurs et éleveurs du Tchad.

\begin{tabular}{|c|c|c|c|c|}
\hline \multicolumn{3}{|c|}{ Type de saison en langues locales } & \multirow{2}{*}{$\begin{array}{l}\text { Appréciations qualitatives } \\
\text { caractéristiques }\end{array}$} & \multirow{2}{*}{ Mois ou périodes } \\
\hline Arabe & Peul & Zimé & & \\
\hline Kharif & Ndungu & N'Dorhilim & $\begin{array}{l}\text { Saison des pluies bien installée, } \\
\text { tous les pâturages sont verts, } \\
\text { abondance de l'eau (mares, } \\
\text { flaques...) }\end{array}$ & Mai à Septembre \\
\hline Darat & Djamde & Wakaria & $\begin{array}{l}\text { Début de saison sèche ou période } \\
\text { de récolte. Les pluies sont rares, et } \\
\text { les points d'eau temporaires } \\
\text { tarissent progressivement. }\end{array}$ & $\begin{array}{l}\text { Septembre à } \\
\text { Novembre }\end{array}$ \\
\hline Chité & Dabbundé & Tersimède & $\begin{array}{l}\text { Saison sèche froide, l'hygrométrie } \\
\text { de l'air est très basse en cette } \\
\text { période. }\end{array}$ & $\begin{array}{l}\text { Novembre à } \\
\text { Février }\end{array}$ \\
\hline Seyf & Ceedu & Terhasse & $\begin{array}{l}\text { C'est la saison sèche chaude. On } \\
\text { note une évaporation très intense ; } \\
\text { la température ambiante augmente } \\
\text { considérablement. Seuls les points } \\
\text { d'eau permanents subsistent et } \\
\text { l'hygrométrique de l'air } \\
\text { augmente. } \\
\text { Les pâturages deviennent épars. } \\
\text { C'est la période de « soudure». }\end{array}$ & Mars à Avril \\
\hline Rouchach & Seeto & $\begin{array}{l}\text { Mbirou } \\
\text { Koygoura }\end{array}$ & $\begin{array}{l}\text { Début de saison des pluies. C'est } \\
\text { la période de pré-hivernage ou } \\
\text { pré-saison des pluies. Arrivée des } \\
\text { premières pluies éparses et } \\
\text { apparition de pousses de premiers } \\
\text { pâturages verts localisés. }\end{array}$ & Avril à Mai \\
\hline
\end{tabular}

ont été confectionnées pour le comptage des fréquences des espèces végétales. Les mesures ont été faites à intervalle régulier, selon 12 strates correspondant chacune à une classe de hauteur de $1 \mathrm{~m}$. Pour mieux distinguer les différentes strates, deux couleurs (rouge et blanche) ont été utilisées. La hauteur de $2 \mathrm{~m}$ a été retenue comme celle au delà de laquelle les feuilles ne sont plus directement accessibles aux ruminants (Hiernaux, 1980 ; Ickowicz, 1995). A chaque point d'observation, le contact d'une espèce avec la perche est réalisé soit par ses feuilles, soit par son tronc ou par ses inflorescences. Une espèce a été recensée une seule fois par point de lecture et par strate. Les paramètres structuraux suivants ont été relevés :
- La fréquence spécifique : c'est le nombre de fois où une espèce considérée apparaît dans une série d'unités d'échantillonnage. C'est le contact d'un organe aérien d'une espèce avec la perche. Il correspond au nombre de contacts de l'espèce le long d'une ligne. C'est une fréquence absolue ;

- la contribution spécifique : elle est définie par le rapport de la fréquence spécifique de l'espèce sur la somme des fréquences spécifiques de toutes les espèces recensées.

\section{Analyse statistique des données}

Le logiciel Excel 2003 a servi à calculer les moyennes, les écarts types et à générer les graphiques d'illustration. Le 
analyses statistiques des distributions des fréquences des espèces par strates et saisons. Pour mettre en évidence les variations saisonnières des espèces, une analyse factorielle des correspondances (AFC) a été réalisée sur deux tableaux de contingence comportant en lignes les espèces, et en colonnes les strates et les saisons.

\section{RESULTATS}

\section{Inventaire des espèces ligneuses}

La densité moyenne des espèces a été de 1248 individus adultes à l'hectare, avec de grandes variabilités (450 à 1624 individus à l'hectare) entre les stations. Le recouvrement des ligneux a été de 60 p.100. Les quatre espèces les plus représentées ont été : Anogeissus leiocarpa, Combretum collinum, Prosopis africana et Pterocarpus erinaceus. Les espèces à distribution régulière ont été $A$. leiocarpa et $C$. collinum. A. leiocarpa a été rencontrée sur l'ensemble des stations échantillonnées, parfois en peuplement quasimonospécifique. La distribution en classe de diamètre des individus traduisant l'état de renouvellement des peuplements ligneux est donnée à la figure 2. Les individus de gros diamètre ont été peu nombreux ou absents dans certains sites.

La régénération a été nulle pour Afzelia africana, Parkia biglobosa, Entada africana, Khaya senegalensis, Stereospermum kunthianum, $P$. erinaceus et $P$. africana. En revanche, la régénération a été bonne chez $A$. leiocarpa, Detarium microcarpum, $C$. collinum et Combretum nigricans. Ces dernières espèces se sont distinguées des autres par une forte densité d'individus jeunes (3200 à 6800 pieds/ha). L'état démographique de jeunes plants est un facteur prépondérant dans la dynamique de la végétation car il détermine le renouvellement des peuplements ligneux (Ouedraogo, 2006).

Variation saisonnière de la disponibilité fourragère ligneuse : distribution des fréquences spécifiques selon les strates

La distribution des fréquences des espèces au niveau des différentes strates a été mise en évidence selon les saisons. On note au niveau de la strate ligneuse de l'ensemble des stations, une variation des fréquences (0 à $53 \%$ ) dans les 12 strates durant les 4 saisons. Pour les strates moyennes et supérieures, la fréquence a été faible pour la majorité des individus. Les strates moyennes ont été dominées par $P$. erinaceus alors que Danielia oliveri a été dominante dans les strates supérieures. Par contre, un bon développement de C. collinum et $C$. glutinosum a été observé au niveau des strates inférieures (1 et $2 \mathrm{~m}$ ) (Figure 3 ). Les fréquences de l'ensemble des strates inférieures (strate 1 et $2 \mathrm{~m}$ ) a très peu varié d'une période à une autre. Les fréquences maximales ont été obtenues dans les strates basses au cours de la saison des pluies : 53,2 \pm 14,4 et $50,0 \pm 12,8$ respectivement pour les

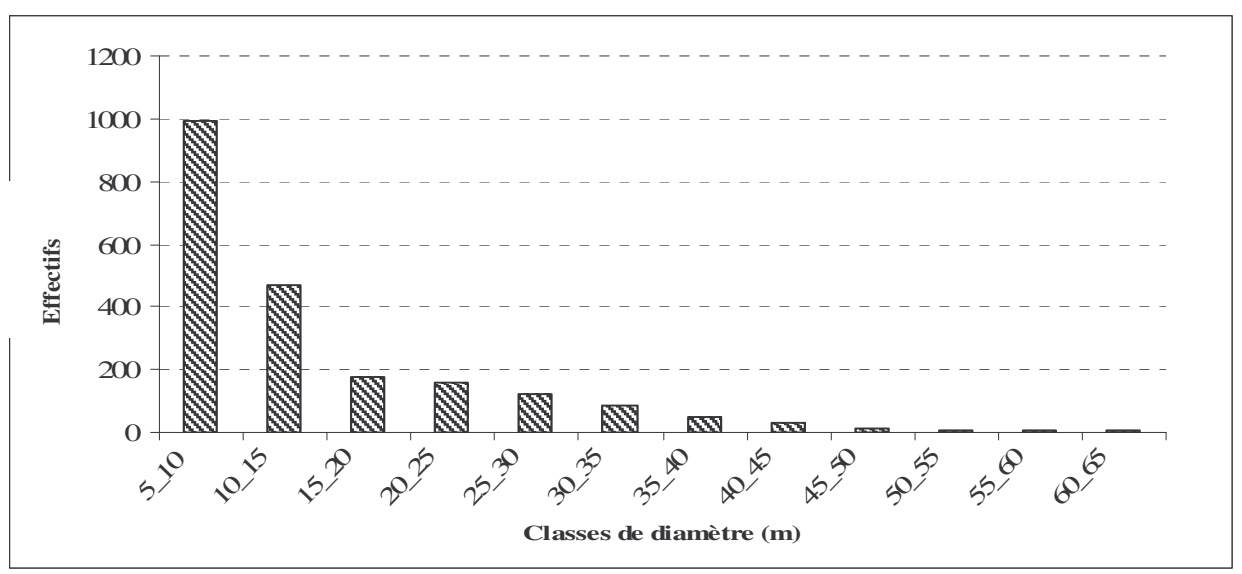

Figure 2 : Structure démographique en fonction des classes de diamètre. 


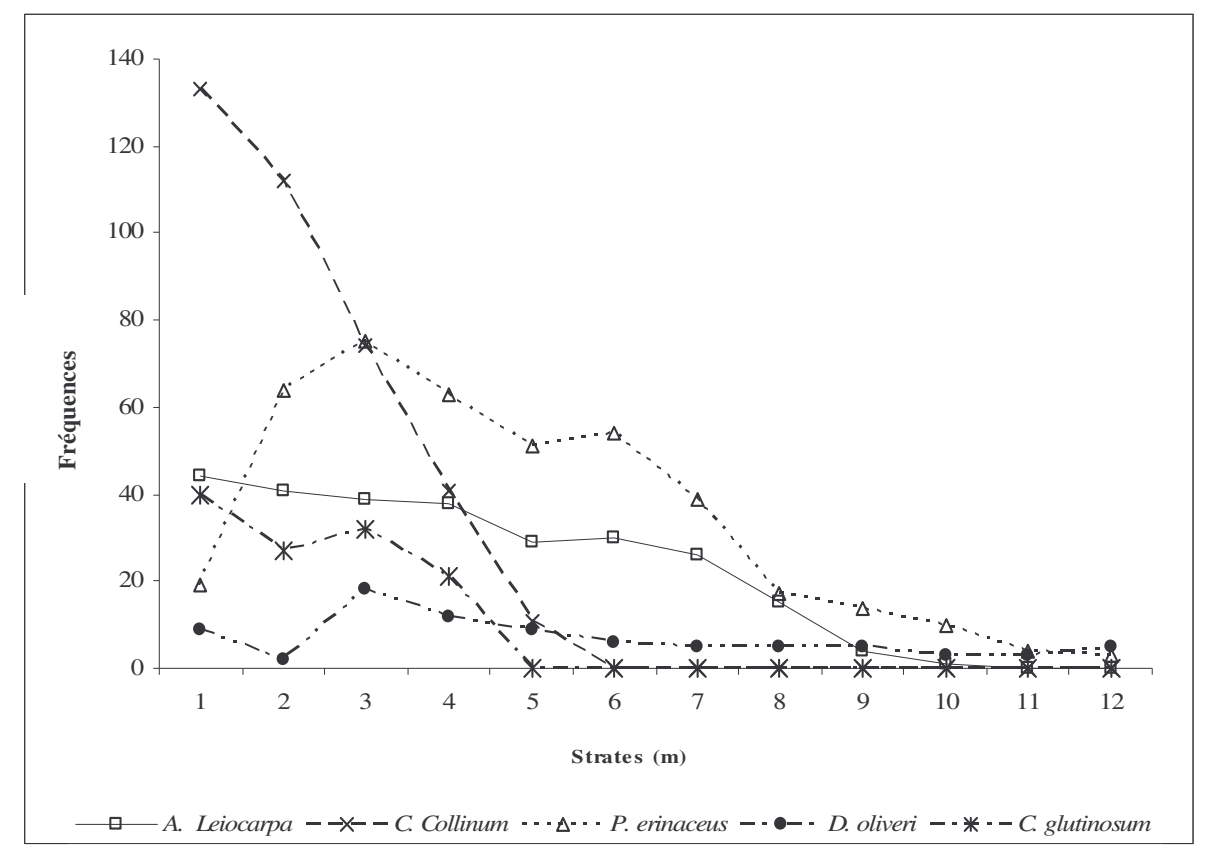

Figure 3: Distribution des fréquences des principales espèces par strate.

strates 1 et $2 \mathrm{~m}$. Ces fréquences ont cependant très peu varié avec les saisons mais ont diminué lorsque le niveau de strates augmente. Elles ont été dans certains cas nulles à partir de la strate 6 m (Figure 4).

\section{Distribution des fréquences spécifiques par strate: analyse factorielle de correspon- dance}

L'essentiel de l'information du tableau de contingence (espèces/strates) est contenu dans les trois principaux axes factoriels. Le plan factoriel constitué des axes 1 et 2 explique près de 84 p.100 de la variance totale. Avec respectivement 32 p.100 et 21 p.100 de variance expliquée, la représentation du premier plan factoriel a montré que positivement, les strates $1 \mathrm{~m}$ et $2 \mathrm{~m}$ interviennent fortement dans la création de l'axe 1 , contrairement à l'axe 2 pour lequel la strate $12 \mathrm{~m}$ possède la plus forte valeur de contribution. Concernant les espèces interceptées par la ligne lors des relevés, $C$. collinum (23 p.100), et D. microcarpum (17 p.100) ont exprimé positivement la plus grande part prise par une espèce donnée dans la variance expliquée par l'axe 1 . D. oliveri (42 p.100) par contre, a contribué fortement à la création de l'axe 2. Les espèces situées aux extrémités des axes sont celles pour lesquelles des fréquences ont été notées uniquement dans les strates inférieures basses et moyennes. Ce sont des espèces arbustives telles que : H. acida, Piliostigma thonningii, C. glutinosum, C. collinum, Sterculia setigera et $D$. microcarpum. La contribution de ces différentes espèces de petite taille explique la séparation des strates 1,2 et $3 \mathrm{~m}$. La position de l'espèce $D$. oliveri placée entre les strates 11 et $12 \mathrm{~m}$ s'explique par la très forte fréquence de l'espèce (62 p.100 et 42 p.100) enregistrée dans ces strates. A. africana et $S$. kunthianum ne sont rencontrées que dans une seule station, chacune avec par ailleurs une faible contribution au recouvrement de l'ordre de 0 à 2 p.100. Elles ont donc été corrélées au facteur 1, mais contribuent faiblement à sa création. Les strates $5,6,7$, et $8 \mathrm{~m}$ se détachent des autres, rassemblées par les fréquences irrégulières et élevées de $A$. leiocarpa et Terminalia avecinioides. $P$. erinaceus contribue à la construction du facteur 1. Cette espèce distingue les strates 9 et $10 \mathrm{~m}$ des autres, par sa fréquence élevée dans ces strates (Figure 5). 


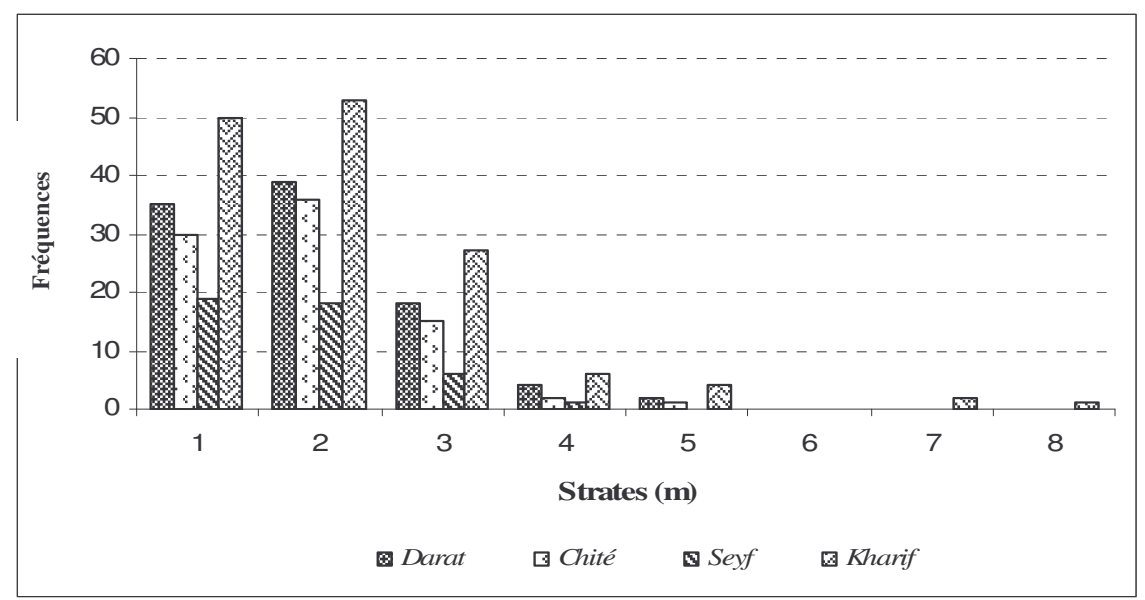

Figure 4 : Distribution des fréquences spécifiques par strate et saison. Darat = Début de saison sèche ; Chité $=$ Saison sèche froide $;$ Seyf $=$ Saison sèche chaude $;$ Kharif $=$ Saison des pluies .

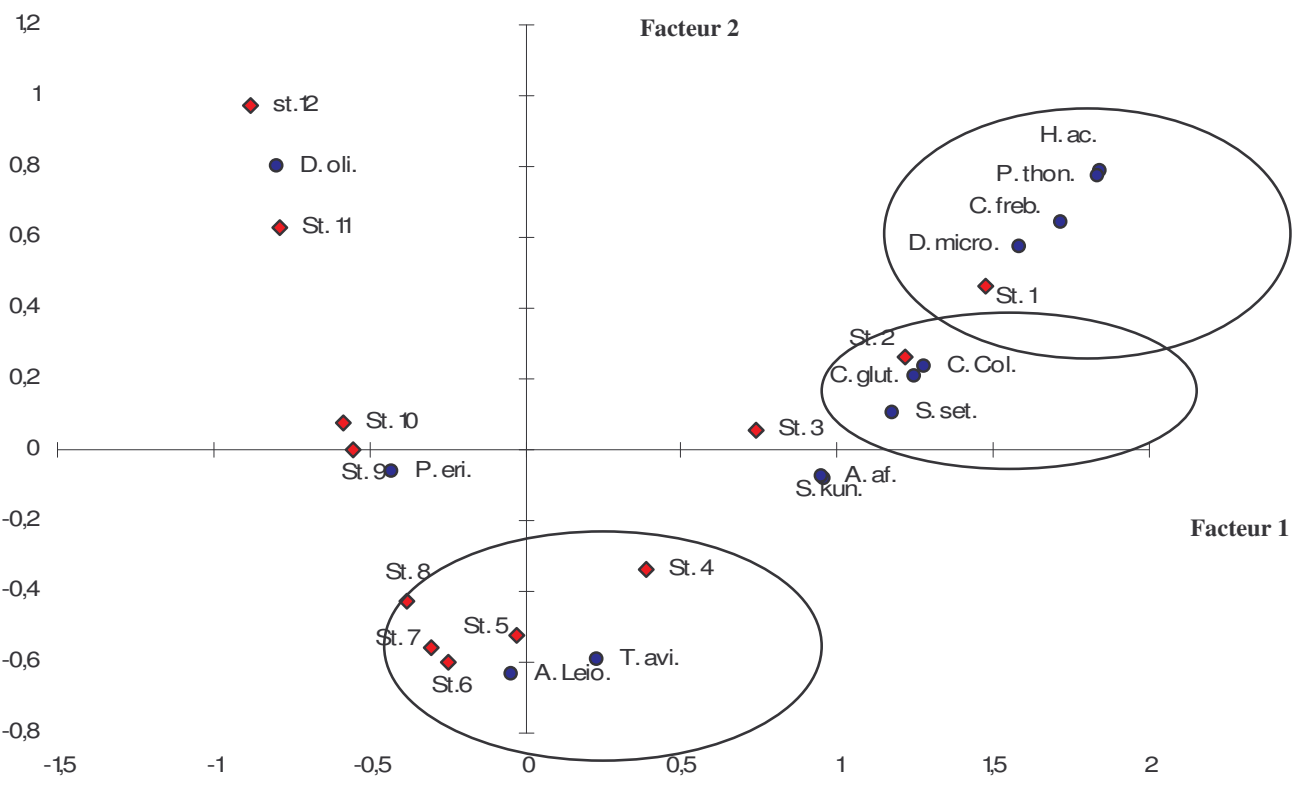

Figure 5: Projection dans le plan des axes 1 et 2 de l'analyse factorielle des correspondances sur les distributions des espèces selon les strates. $P$. thon. $=$ P. thonningii; C. feb. $=$ Crossopterix febrifuga $;$ D. micro. $=$ D. microcarpum $;$ C. col. $=$ C. collinum $;$ C. glu.$=C$. glutinosum $; S$. set. $=S$. setigera $; A$. af. $=$ A. africana $; S$. kun. $=S$. kunthianum $;$ T. avi. $=$ Terminalia avecinioides $;$ A. leio. $=$ A. leiocarpa $;$ P.eri. $=$ P. erinaceus $;$ D.oli. $=$ D. oliveri $;$ H. ac . $=$ H. acida . St. $=$ Strate

Variation saisonnière de la disponibilité fourragère ligneuse : distribution des fréquences spécifiques selon les saisons

Une chute considérable des fréquences a été observée entre la saison pluvieuse et la saison sèche chaude pour l'ensemble des espèces. La fréquence maximale est obtenue dans les strates les plus basses pendant la saison des pluies. La comparaison des fréquences totales, espèce par espèce, a 
montré qu'avec $C$. collinum, on enregistre le plus grand nombre de fréquence $(30,5 \pm 6,0)$ en saison sèche chaude, bien que cette saison soit celle au cours de laquelle le moins de contacts soit noté pour toutes les espèces et quelque soit le niveau de la plante (Figure 6). Pendant la saison des pluies, les plus fortes valeurs $(26,4 \pm 5,0)$ ont été obtenues avec $P$. erinaceus. Plusieurs individus se sont distingués par leur faible fréquence pendant toutes les saisons: $C$. febrifuga $(0,85 \pm 0,5), A$. africana $(0,85 \pm 0,5), H$. acida $(3,8 \pm 1,4), D$. oliveri $(4,8 \pm 1,2)$ et $M$. kerstingii $(0,85 \pm 0,1)$. A. africana a une fréquence nulle en saison sèche chaude et très faible pendant les 3 autres saisons. C. glutinosum a aussi une faible distribution durant toutes les saisons à l'exception du début de saison sèche où il a une fréquence moyenne de 17,0 $\pm 6,1$ (Figure $6)$.

Distribution des fréquences spécifiques selon les saisons: analyse factorielle de correspondance

L'analyse factorielle des correspondances a été effectuée à partir des relevés des contributions spécifiques des espèces sur l'ensemble des sites. La totalité de l'information contenue dans le tableau de contingence espèces/saisons est résumée avec les trois axes. Les 2 premiers axes factoriels (plan factoriel 1-2) expliquent 93 p.100 de la variance totale. Les distributions des fréquences ont été fortement liées aux caractéristiques des différentes saisons (Figure 7).

Le début de saison sèche (68 p.100) et la saison sèche chaude $(25 \mathrm{p} .100)$ ont contribué fortement à la détermination du facteur de l'axe 1. Le début de saison sèche est éloigné de l'axe 2 à cause de la contribution de $C$. glutinosum. Cette espèce est d'autant plus importante dans cette analyse que sa contribution spécifique maximale (17 p.100) a été observée pendant cette même saison. L'axe 2 explique par contre 26 p.100 de la variance totale et a distingué une forte contribution de la saison des pluies (57 p.100). Ce plan factoriel met également en évidence la faible fréquence des espèces telles que Crossopterix febrifuga, Hymenocardia acida, Monotes kerstingii et A. africana. Elles présentent une très faible contribution spécifique et sont parfois absentes sur certaines lignes. Ces espèces ne présentent dans certaines stations aucun contact pendant la saison sèche chaude. Cette saison se détache de l'ensemble des autres saisons par les forts contacts enregistrés sur $S$. kunthianum $(8,33 \pm 16,6)$ et $D$. microcarpum $(22,1 \pm 19,6)$ en cette période. Par contre $C$. collinum présente à peu près la même contribution en début de saison sèche, en saison sèche froide et en saison sèche chaude (Figure 7).

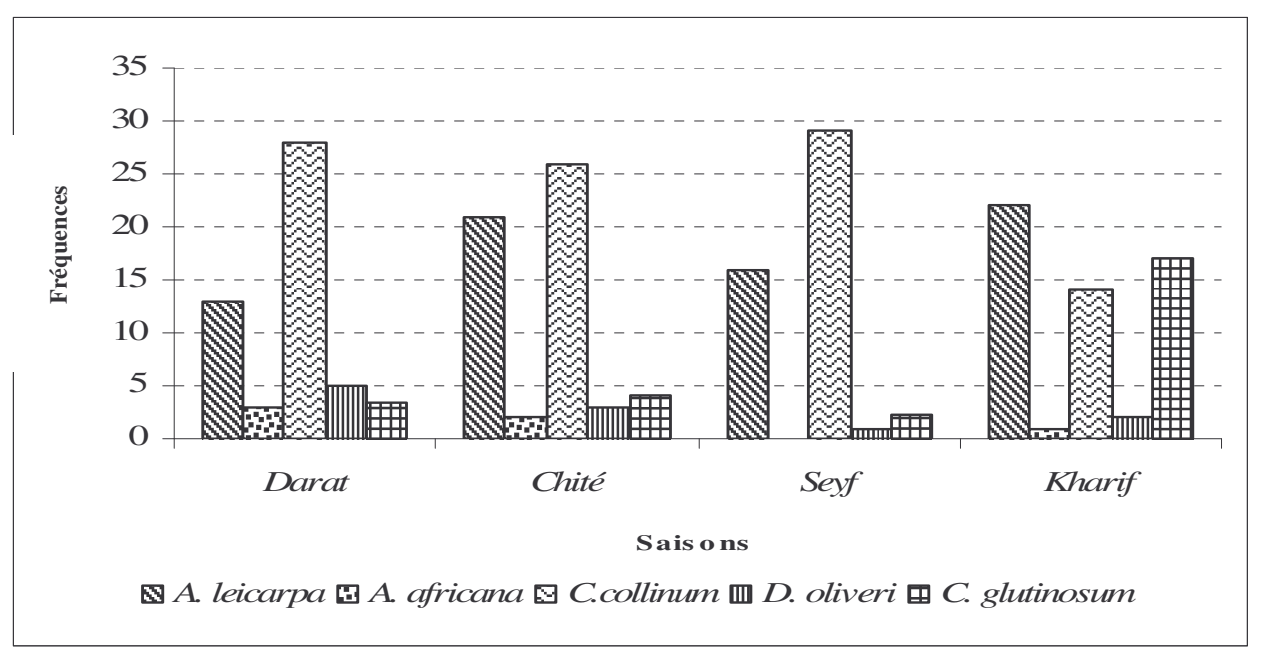

Figure 6 : Distribution des fréquences des principales espèces selon les saisons. Darat $=$ Début saison sèche $;$ Chité $=$ Saison sèche froide $;$ Seyf $=$ Saison sèche chaude $;$ Kharif $=$ Saison des pluies . 


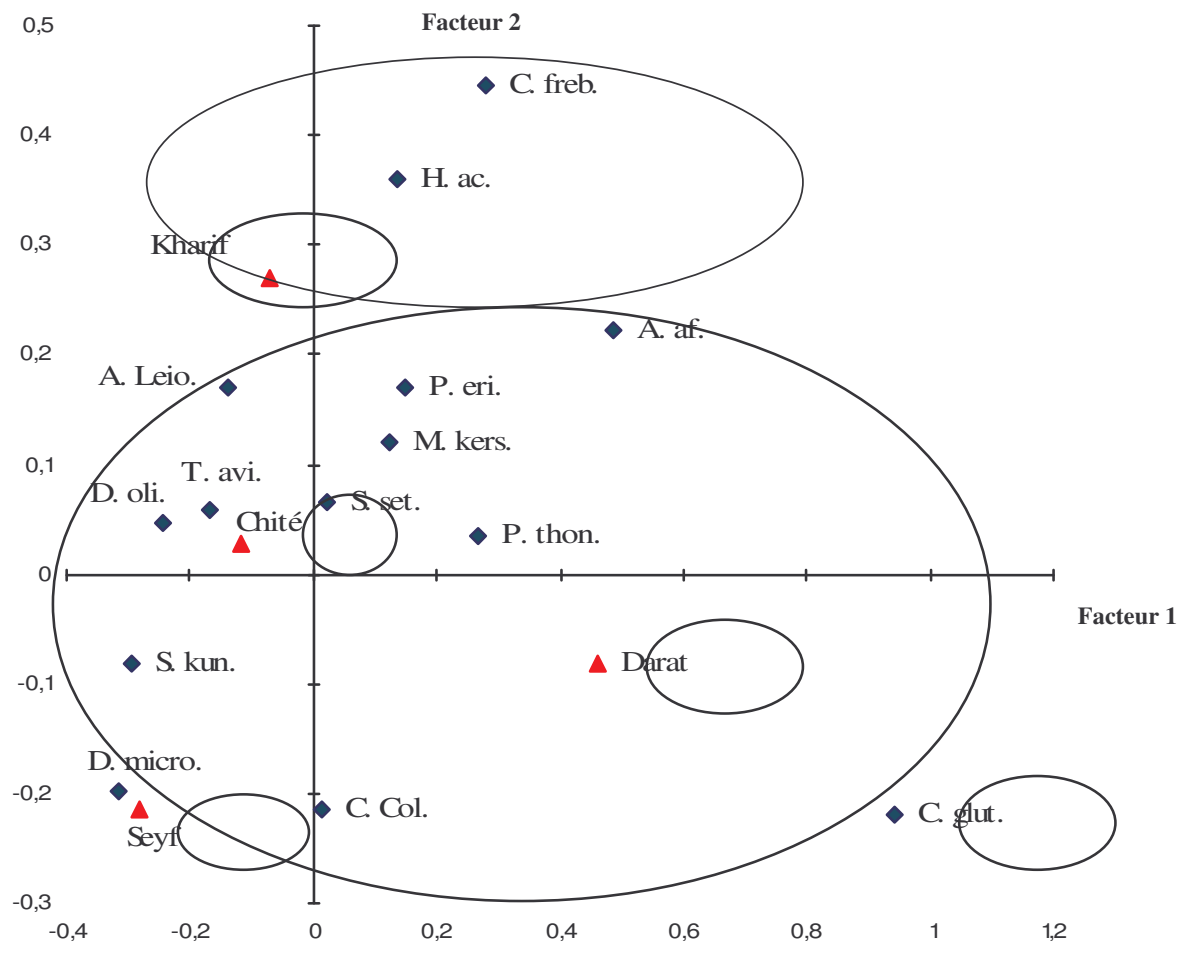

Figure 7: Projection dans le plan factoriel 1 et 2 de l'analyse factorielle des correspondances sur la distribution des espèces selon les saisons. Darat $=$ Début saison sèche $;$ Chité $=$ Saison sèche froide $;$ Seyf $=$ Saison sèche chaude $;$ Kharif $=$ Saison des pluies $; P$. thon.$=P$. thonningii; $C$. feb. $=C$. febrifuga $; D$. micro. $=$ D. microcarpum ; C. col. $=$ C. collinum $;$ C. glu.$=$ C. glutinosum $;$ S. set. $=S$. setigera $;$ A. af. $=$ A. africana $; S$. kun. $=S$. kunthianum $;$ T. avi . $=$ T. avecinioides $;$ A. leio. $=$ A. leiocarpa $;$ P.eri. $=$ P. erinaceus $;$ D.oli. $=$ D. oliveri $; H$. ac. $=H$. acida.

\section{DISCUSSION}

Les stations dans lesquelles s'est effectué notre échantillonnage ont été choisies pour illustrer au mieux la situation globale dans notre zone géographique d'étude. Bien typées sur le plan floristique, leur représentativité en termes de superficie et d'utilisation pastorale nous a permis de décrire leur évolution pendant les quatre (4) saisons selon le calendrier fourrager des populations pasteurs, même si ce type de découpage de l'année commence à montrer ses limites dans un environnement sahélien soumis entre autres à la pression croissante et néfaste des aléas climatiques. Cet échantillonnage a mis en évidence une variation dans le nombre de contacts et dans la distribution des fréquences enregistrées, à la fois en fonction des strates et des saisons. Plusieurs raisons peuvent expliquer la baisse des fréquences observée, car cette situation n'est pas seulement liée à la phénologie des espèces. Les diminutions des fréquences enregistrées dans les strates moyennes et supérieures chez $C$. febrifuga, A. africana, $H$. acida, D. oliveri et $M$. kerstingii pendant la saison des pluies et le début de saison sèche pourraient s'expliquer par les coupes de bois, les feux de brousse ou encore des coupes pour la complémentation du régime des petits ruminants au piquet pendant cette saison. Le développement et la phénologie des ligneux n'ont pas ce caractère de dépendance vis-à-vis de la saison des pluies (Piot, 1980). La pression exercée sur A. africana, $H$. acida et $D$. oliveri, très sollicitées en saison sèche chaude et en début de saison sèche, explique leur disparition complète des strates plus basses (pâturage aérien) et hautes (coupe de bois, émondage). Le nombre de contacts 
élevés observés pendant le début de saison sèche et la saison sèche froide chez $C$. collinum, C. glutinosum et A. leiocarpus ont constitué le signe d'une faible pression pastorale et d'une bonne régénération pendant ces saisons. D'ailleurs ces espèces ne subissent pas la même pression pastorale que les précédentes parce qu'elles n'ont pas le même intérêt fourrager. Le pâturage aérien est très peu pratiqué en début de saison sèche et en saison sèche froide à cause de l'abondance des pâturages herbacés d'une part et, d'autre part, des champs qui offrent une disponibilité élevée en résidus de culture exploités en vaine pâture par les animaux (Lhoste, 1986). Les fréquences ont été par contre faibles en saison sèche chaude, en raison du pâturage aérien pratiqué en cette période de déficit alimentaire. Les espèces les plus concernées par cette pratique ont été : $K$. senegalensis, $A$. africana, $P$. africana et $P$. erinaceus. Ces espèces ont été aussi citées par Petit et Mallet (2000) comme celles qui sont les plus émondées dans le terroir de Kourouma en saison sèche, en zone soudanienne du Burkina Faso. Cette diminution observée dans les fréquences en cette période dite «de soudure » s'expliquerait également par la phénologie des espèces. En effet, les différents stades phénologiques des espèces sont corrélés avec les paramètres climatiques et les textures du sol (Mahamane et al., 2007).

La saison sèche chaude correspond à une période morte au cours de laquelle la population s'adonne à d'autres activités rémunératrices (coupes de bois, fabrication de charbon, chasse, défriches...), qui ne sont pas sans conséquence sur les peuplements ligneux. Chez les espèces ligneuses d'une manière générale, la feuillaison commence avant ou au début de la saison des pluies. Quant à la chute des feuilles, elle débute plus ou moins tard en saison sèche, souvent bien après la dessiccation des herbacées. Cette défoliation périodique se traduit par d'importantes fluctuations saisonnières du disponible et de la qualité des fourrages offerts par chacune des composantes des parcours naturels (Hiernaux et al., 1994).

Notre étude a mis en évidence le comportement particulier de $C$. collinum, dont la fréquence a été importante pendant la saison sèche chaude. Cette espèce a une phénologie caractérisée par une feuillaison assez étalée dans le temps (Hiernaux et al., 1994). Par l'étalement de son cycle végétatif, cette espèce contribue efficacement à la pondération des déficits alimentaires saisonniers rendant un peu moins sévères les conditions d'élevage en saison sèche. Une distribution des individus selon leur diamètre en forme d'histogramme en « $\mathrm{L}$ » de tendance exponentielle est notée, indiquant une forte proportion de jeunes et un fort potentiel de reconstitution des peuplement stables (Ouedraogo et al., 2006).

Il a été expérimentalement établi que le mode, le rythme et l'époque d'exploitation influent sur le peuplement ligneux (Cisse, 1980b ; Cisse, 1984). La surexploitation des espèces ligneuses, associée à la sévérité du climat et aux feux de brousse très fréquents, affecte considérablement leur possibilité de régénération. L'exploitation pastorale peut également influer la dynamique et la production des ligneux. Une pâture trop permanente empêche certaines espèces ligneuses de fructifier. Elle contribue aussi à l'élimination systématique des jeunes repousses. La production foliaire peut être également stimulée ou déprimée selon les espèces, la saison et l'intensité de l'effeuillage (Cisse, 1980a ; Cisse, 1980b). Les mêmes sources notent que la coupe n'a aucun effet sur la densité maximale du feuillage mais modifie en revanche profondément le rythme de feuillaison.

Dans notre zone d'étude, la gestion traditionnelle des fourrages ligneux par émondage, élagage ou effeuillage empêche la production des semences. $\mathrm{Si}$ la situation perdure, le vieillissement du peuplement et sa disparition seront inéluctables. L'émondage lorsqu'il ne provoque pas la mort de l'arbre, tend à étaler la saison de feuillaison en retardant la chute des feuilles (Hiernaux et al., 1994) au point que les rejets, qui se maintiennent à l'état végétatif, restent parfois feuillés à contre-saison (Cisse, 1984). La présente étude a été réalisée dans un milieu où l'élevage est de type extensif, ce qui limite les possibilités de contrôle des pressions d'exploitation sur les ressources fourragères. La zone étant soumise à une forte contrainte anthropique, on pourrait assister à la disparition de nombreuses espèces 
surexploitées si aucune mesure de gestion n'est prise. Dans la gestion des ressources fourragères (ligneuses et herbacées), les principales questions qui se posent sont la protection dès la régénération et l'amélioration de la production de la biomasse (Onana et Devineau, 2002). La mise en œuvre des principes de gestion des espèces fourragères a fait l'objet de nombreuses études (Iemvt-Cirad, 1989). Ces principes tiennent de : (i) l'émondage différé avec des intervalles de temps de repos, (ii) l'interdiction des pâtures sous les portegraines, et (iii) l'interdiction ou le contrôle de la pratique des feux de brousse. Afin de permettre la régénération des espèces, la mise en repos temporaire du terroir s'impose. Néanmoins, dans le contexte social actuel, ces différentes mesures indispensables à une bonne régénération, ne sont pas faciles à appliquer du fait de l'inorganisation de la gestion des parcours, de la réduction des espaces agro-sylvo-pastoraux et de la pression démographique (humaine et animale) croissante.

\section{Conclusion}

L'étude a mis en évidence l'importance des ressources fourragères ligneuses dans l'alimentation du bétail en élevage extensif. Le lien de la disponibilité saisonnière de ces ressources avec la pression de pâture, la phénologie des espèces ou la pression anthropique est démontré. L'étude montre également l'existence des contraintes alimentaires pendant les périodes d'abondance et une réaction du pâturage naturel (production, recouvrement et diversité) face aux contraintes environnementales. Toutes ces informations permettent d'élaborer des mesures de gestion de l'espace pastoral et une meilleure valorisation des ressources fourragères ligneuses du terroir. Toutefois, les observations faites sur une année ne permettent pas d'appréhender les fluctuations interannuelles indispensables pour valider ce travail et mieux apprécier la situation. De plus, l'étude gagnerait à être complétée par des observations du poids et de l'état corporel des animaux du terroir en lien avec les fluctuations saisonnières des ressources fourragères.
REFERENCES BIBLIOGRAPHIQUES

Breman H, De Ridder N. 1991. Manuel sur les Pâturages des Pays Sahéliens. ACCTCTA. KARTHALA: Paris.

Cabot J. 1965. Le Bassin du Moyen Logone. ORSTOM.

César J, Zoumana C. 1999. Les régimes alimentaires des bovins, ovins et caprins dans les savanes de Côte-d'Ivoire et leurs effets sur la végétation. Fourrages., 159: 237-252.

Cisse MI. 1980a. Production fourragère de quelques arbres sahéliens : relation entre biomasse foliaire maximale et divers paramètres physiques. In Les Fourrages Ligneux en Afrique. Etat Actuel des Connaissances, Le Houérou HN (éd). CIPEA : Addis Abeba; 203-208.

Cisse MI. 1980b. Effet de divers régimes d'effeuillage sur la production foliaire de quelques buissons fourragers dans la zone soudano-sahélienne. In Les Fourrages Ligneux en Afrique. Etats Actuel des Connaissances, Le Houérou HN (éd). CIPEA: Addis Abeba; 209-212.

Cisse MI. 1984. Synthèse des essais d'ébranchages pratiqués sur quelques arbres fourragers sahéliens de 1978 à 1983. CIPEA : Bamako Mali, p.18.

Clanet JC. 1975. Les éleveurs de l'Ouest tchadien. La mobilité des éleveurs du Kanem et leur réponse à la crise climatique de 1969-1970. Thèse de Doctorat de 3è cycle, Université de Rouen, P. 198.

Daget P, Poissonet J. 1971. Une méthode d'analyse phytologique des prairies. Critères d'application. Ann. Agron., 22: 5-41.

Gaston A. 1981. La Végétation Pastorale du Bassin du Lac Tchad. In Atlas d'élevage $d u$ Bassin $d u$ Lac Tchad. CIRADEMVT : Wageningen, CTA, Montpellier; 39-55.

Hernaux P, Cisse MI, Diara L, De Leeuw PN. 1994. Fluctuations saisonnières de la feuillaison des arbres et des buissons sahéliens. Conséquences pour la quantification des ressources fourragères. Revue Elev. Méd. Vét. Pays Trop., 47: 117-125.

Hiernaux P, Le Houerou HN. 2006. Les parcours du Sahel. Sécheresse, 17: 51-71. 
Hiernaux P. 1980. Inventaire du potentiel fourrager des arbres et arbustes d'une région du Sahel malien. Méthodes et premiers résultats. In Les Fourrages Ligneux en Afrique. Etat Actuel des Connaissances, Le Houerou HN (éd). CIPEA: Addis Abeba, Ethiopie; 195-201.

Hiernaux P. 2000. Implication of the « New Rangeland Paradigm» for natural ressource management. In The Sahel: Energy Supply, Economic Pillars of Rural Sahelian Communities, Need for Revised Development Strategies, Adriansen $\mathrm{H}$, Reenber A, Nielsen I (eds). Copenhagen, Danemark; 231-245.

Ickowicz A. 1995. Approche dynamique du bilan fourrager appliqué à des formations pastorales du Sahel tchadien. Thèse de Doctorat de l'Université de Paris XII, p. 471.

IEMVT-CIRAD 1989. Les ligneux fourragers et fruitiers en zones tropicales et subtropicales. Paris, France, Ministère de la Coopération et du Développement, Maison-Alfort, France, Iemvt-Cirad, fiche technique d'élevage tropicale $\mathrm{n}^{\circ} 3.8 \mathrm{p}$.

IEMVT-CIRAD 1989. Les ligneux fourragers et fruitiers en zones tropicales et subtropicales. Paris, France, Ministère de la Coopération et du Développement, Maison-Alfort, France, Iemvt-Cirad, fiche technique d'élevage tropicale ${ }^{\circ} 6.8 \mathrm{p}$.

IEMVT-CIRAD 1989. Les ligneux fourragers et fruitiers en zones tropicales et subtropicales. Paris, France, Ministère de la Coopération et du Développement, Maison-Alfort, France, Iemvt-Cirad, fiche technique d'élevage tropicale $\mathrm{n}^{\circ} 10.8 \mathrm{p}$.

Le Houerou HN. 1980. Le rôle des ligneux dans les zones sahéliennes. In Les Fourrages Ligneux en Afrique. Etat Actuel des Connaissances, Le Houerou HN (éd). CIPEA: Addis Abeba, Ethiopie; 85-101.
Lhoste P. 1986. L'Association AgricultureElevage. Evolution du Système AgroPastoral au Sine Saloum (Sénégal). IEMVT, Maison Alfort; p. 314.

Mahamane A, Saadou M, Lejoly J. Phénologie de quelques espèces ligneuses du parc de «W $»$ (Niger). Sécheresse, 18: 354-358.

Onana J, Devineau JL. 2002. Afzelia africana Smith ex Persoon dans le NordCameroun. Etat actuel des peuplements et utilisation pastorale. Revue Elev. Méd. Pays Trop., 55: 39-45.

Ouedraogo A, Hahn-Hadjali K, Thiombiano A, Guinko S. 2006. Diagnostic de l'état de dégradation des peuplements de quatre espèces ligneuses en zone soudanienne du Burkina Faso. Sécheresse, 17: 485-491.

Ouedraogo A. 2006. Diversité et dynamique de la végétation ligneuse de la partie orientale du Burkina Faso. Thèse de Doctorat Thèse de Doctorat de l'Université de Ouagadougou, p.195.

Petit S, Mallet B. 2000. L'émondage des arbres fourragers : détail d'une pratique. Bois et forêts des Tropiques, 270: 35-45.

Piot J. 1980. Les méthodes de gestion et d'exploitation des fourrages ligneux : peuplements naturels et plantations artificielles. In Les Fourrages Ligneux en Afrique: Etat Actuel des Connaissances. Le Houerou HN (éd). CIPEA: Addis Abeba, Ethiopie; 335-343.

Rondeux J. 1999. La Mesure des Arbres et des Peuplements ( $2^{\text {è }} \quad$ Edn). Presses Agronomiques : Gembloux, Belgique.

Sacko B. 1991. Caractérisation des Disponibilités Fourragères Ligneuses sur des Parcours Naturels Sahéliens Exploités par des Bovins, Ovins ou Caprins. ISRA, IERSERZ, p.100. 